\title{
Uso de geotecnologias integradas a um sistema de Informações Geográficas (SIG) na análise da situação ambiental da região do parque estadual do Jabre/PB
}

O crescimento populacional e as atividades antrópicas sem planejamento têm causado significativas perdas socioambientais no mundo todo, sendo tema frequente em muitas regiões brasileiras. O Parque Estadual do Jabre está inserido nos municípios paraibanos de Maturéia e Mãe D'água e apresenta fatores socioambientais característicos, além de elementos ambientais comuns a região semiárida, no entanto, é uma área que requer atenção especial no desenvolvimento de ações que visem preservar e mitigar os inúmeros problemas presentes. Este estudo objetiva identificar características altimétricas da região por meio de Geotecnologias, utilizando ferramentas como arquivos shapefile e Modelos Digitais de Elevação (MDE), além de analisar a alteração do uso do solo nos municípios de Maturéia e Mãe D'água nos últimos 30 anos através de imagens dos satélites Landsat 4-5 e Sentinel 2. As questões metodológicas foram integradas em ambiente de Sistema de Informações Geográficas (SIG) com apoio de dados Navigation Satellite System (GNSS) para uma identificação mais precisa dos elementos do meio. Os resultados mostraram que a área de estudos apresenta elementos topográficos que favorecem processos erosivos, bem como eventos relacionados à vulnerabilidade social, também se visualizou a alteração do uso e ocupação do solo ao logo dos últimos 30 anos. Além disso, os resultados permitiram a disponibilidade de dados ambientais que poderão ser utilizados como suporte no planejamento e gestão territorial. Este estudou demonstrou ainda, que as abordagens metodológicas baseadas no uso de Geotecnologias podem subsidiar o gerenciamento local e reduzir os gastos públicos com situações emergenciais.

\section{Use of geotechnologies integrated to a Geographic Information System (GIS) in the environmental situation analysis of the Jabre/PB state park region}

\begin{abstract}
Population growth and unplanned anthropogenic activities have caused significant social and environmental losses worldwide, being a frequent theme in many Brazilian regions. The Jabre State Park is located in the Paraiban municipalities of Maturéia and Mãe D'água and has characteristic socioenvironmental factors, in addition to environmental elements common to the semiarid region. However, it is an area that requires special attention in the development of actions aimed at preserving and mitigate the numerous problems present. This study aims to identify the altimetric characteristics of the region through geotechnologies, using tools such as shapefile files and Digital Elevation Models (MDE), as well as analyzing the land use change in the municipalities of Maturéia and Mãe D'água over the last 30 years through Landsat 4-5 and Sentinel 2 satellite images. Methodological issues have been integrated into a Geographic Information System (GIS) environment supported by Navigation Satellite System (GNSS) data for more accurate identification of the environment elements. The results showed that the study area presents topographic elements that favor erosive processes, as well as events related to social vulnerability, and also the alteration of land use and occupation over the last 30 years. In addition, the results allowed the availability of environmental data that can be used as support in territorial planning and management. This study also demonstrated that methodological approaches based on the use of geotechnologies can support local management and reduce public spending on emergency situations.
\end{abstract}

Keywords: Geotechnology; Remote sensing; Land use and occupation.

Priscilla Gomes Barbosa (D)

Universidade Estadual da Paraíba, Brasil http://lattes.cnpq.br/4823633249815596 http://orcid.org/0000-0002-3054-5680 priscillagomes.engambiental@gmail.com

Alan Barbosa Cavalcanti

Universidade Estadual da Paraíba, Brasil http://lattes.cnpq.br/5910031792518971 profalancavalcanti@gmail.com

João Hugo Baracuy da Cunha Campos (it) Universidade Estadual da Paraíba, Brasil http://lattes.cnpq.br/2677113447182816 http://orcid.org/0000-0002-4796-0696 joaohugo@uepb.edu.br

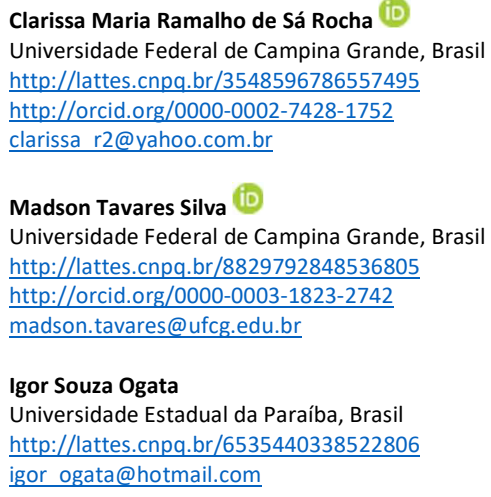

Leonardo Medeiros da Costa

Universidade Estadual da Paraíba, Brasil http://lattes.cnpq.br/3404728721924506 leonardom.costa@yahoo.com.br

\section{Alice Oliveira Justo}

Universidade Estadual da Paraíba, Brasil

http://lattes.cnpq.br/1855338170520912 alicejusto27@gmail.com

Gilmara Rayanne da Silva Chagas

Universidade Estadual da Paraíba, Brasil http://lattes.cnpq.br/0765309614262447 gilchagas150@gmail.com

\section{Referencing this:}

SOBRENOME, N. N.; SOBRENOME, N. N.. Uso de geotecnologias integradas a um sistema de Informações Geográficas (SIG) na análise da situação ambiental da região do parque estadual do Jabre/PB. Revista Ibero Americana de Ciências Ambientais, v.10, n.6, p.146153, 2019. DOI: http://doi.org/10.6008/CBPC2179- 
Uso de geotecnologias integradas a um sistema de Informações Geográficas (SIG) na análise da situação ambiental da região do parque estadual

\section{INTRODUÇÃO}

O crescimento e a concentração da população e das atividades humanas em muitas circunstâncias, com deficiências de planejamento, conjuntamente com o manejo ambiental deficiente e a ocorrência de desmatamentos e avanços excessivos de algumas culturas agrícolas pode gerar territórios vulneráveis (GRECO, 2015). Em sua natureza plural de inúmeras facetas e dimensões, a quantificação, a qualificação e a espacialização da vulnerabilidade de uma região são extremamente complexas, mas de fundamental importância (SZLAFSTEIN, 2015). Consideram-se territórios vulneráveis os que apresentam exposição elevada e duradoura a riscos e ameaças que, pela sua gravidade, exigem intervenções coordenadas, envolvendo diferentes atores e distintos instrumentos de política.

O semiárido brasileiro possui características naturais que o tornam susceptível à erosão e consequentemente, a vulnerabilidade territorial: vegetação nativa esparsa, solos pouco desenvolvidos e escassez hídrica com chuvas concentradas em um curto período de tempo (PEREIRA et al., 2014). O atendimento às demandas hídricas é a questão mais discutida na região, tanto cientificamente, como politicamente, devido à necessidade de abastecimento humano e atendimento aos usos múltiplos indispensáveis ao desenvolvimento de algumas atividades econômicas. Contudo, é importante ressaltar que os componentes de uma bacia hidrográfica interagem entre si e estão interligados, de forma que alterações na qualidade do solo refletirão também na qualidade e quantidade da água (MIRHOSSEINI et al., 2018).

Conhecer a vulnerabilidade natural e ambiental de uma região e estudar a resposta dos atributos físicos e químicos do solo ao uso e ocupação, pode servir como um "sistema de alerta precoce" (AQUINO et al., 2017) que auxilia os cientistas e o poder público nas tomadas de decisão sobre o uso e manejo adequado do solo e na seleção de áreas prioritárias de conservação e/ou preservação da geodiversidade e recuperação do solo, contribuindo para a manutenção da qualidade ambiental e para a implementação de políticas ambientais (CUIABANO et al., 2017; BÜNEMANN et al., 2018; MEHRA et al., 2018).

A vulnerabilidade dos ambientes em função de suas características naturais e do uso e ocupação do solo podem ser avaliadas a partir da análise integrada de aspectos físicos e ambientais, por meio de Sistemas de Informações Geográficas (SIG's) (ZHAO et al., 2018). Neste sentindo, à elaboração de dados capazes de fornecer subsídio à análise integrada da vulnerabilidade em regiões serranas é um instrumento essencial no planejamento e gestão ambiental de territórios vulneráveis. Nesta perspectiva, objetiva-se com esse estudo analisar as alterações relacionadas ao uso e ocupação do solo por meio de geotecnologias no Parque Estadual do Jabre/PB, para que de acordo com os resultados obtidos, sejam criadas ações que poderão auxiliar futuras medidas de proteção ambiental.

\section{MATERIAIS E MÉTODOS}

O Parque Estadual do Jabre está localizado entre os municípios paraibanos de Mãe d'água e Maturéia (Figura 1), apresenta um dos ecossistemas mais ricos do estado, abrigando árvores típicas da mata atlântica e elementos da caatinga. Localizado na microrregião da Serra do Teixeira, apresenta entre outras 
características grandes elevações que contribuem para a manutenção da dinâmica ambiental local.

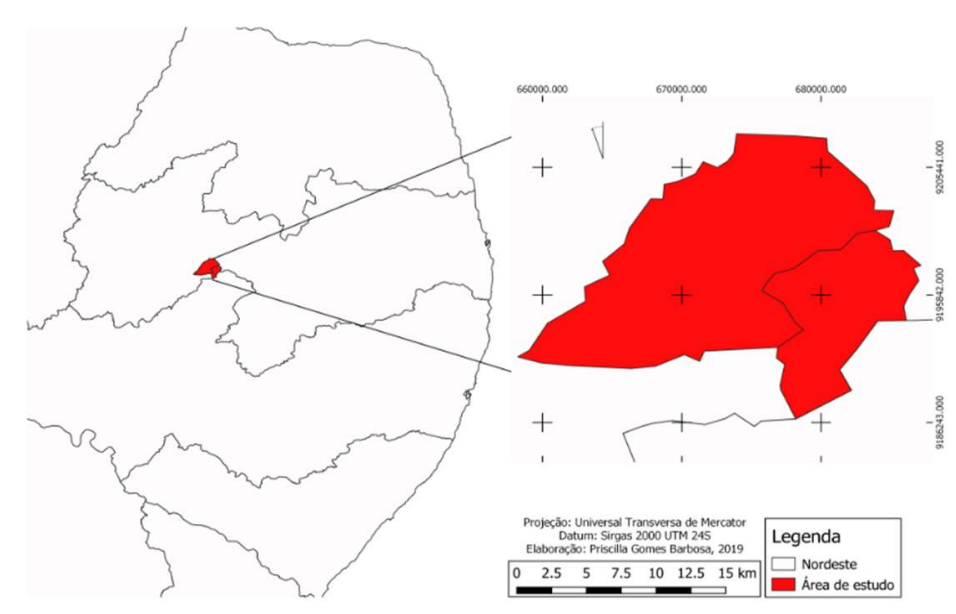

Figura 1: Área de estudo.

Para execução do trabalho foi necessária à obtenção de informações referentes às características da área de estudo através de: 1) Arquivos shapefile disponibilizados pela Agência Executiva de Gestão das Águas da Paraíba (AESA); 2) Dados referentes ao Modelo Digital de Elevação disponibilizados pelo Instituto Nacional de Pesquisas Espaciais (INPE) do SRTM (Shuttle Radar Topography Mission); 3) Dados matriciais (raster) imagem multiespectral do satélite Landsat 4-5 (215/65) com cena do dia 24/10/1999 e dados matriciais Sentinel 2 (215/65) com cenas do dia 03/10/2019 disponíveis gratuitamente na base de dados da USGS (United States Geological Survey - Serviço Geológico dos Estados Unidos).

A primeira parte deste trabalho consistiu na delimitação dos municípios que compõe o Parque Estadual do Jabre por meio de arquivo shapefile disponibilizado pela Agência Executiva de Gestão das Águas da Paraíba (AESA), a manipulação dos dados e extração das informações foi realizada no software livre Qgis 2.18. Em seguida, obteve-se os dados referentes à declividade e relevo da área de estudo, através de arquivo matricial contendo os dados pertencentes ao Modelo Digital de Elevação.

Tabela 1: Classes escolhidas como elementos importantes neste estudo.

\begin{tabular}{|l|l|}
\hline CLASSE & COLORAÇÃO \\
\hline Água & Azul \\
\hline Vegetação & Verde \\
\hline Área construída & Vermelho \\
\hline Agricultura & Amarelo \\
\hline Solo exposto & Cinza \\
\hline
\end{tabular}

O mapa de uso do solo foi obtido via imagem multiespectral disponibilizada pelos satélites Landsat 4-5 sensor ThematicMapper - TM, com resolução espacial de 30 metros e Sentinel 2 com resolução espacial de $20 \mathrm{~m}$, que podem ser adquiridas gratuitamente pelo USGS, e envolveu inicialmente a agregação das bandas multiespectrais disponíveis e posterior recorte da imagem para área de interesse, e criou-se uma camada de treinamento, onde realizou-se a classificação supervisionada dos pixel da imagem indicado os elementos de imagem presentes naquela cena, considerando para isto, características vistas como importantes (Tabela 1) 
neste tipo de análise, possibilitando deste modo, a visualização espacial das interações que ocorrem na região estudada.

\section{RESULTADOS E DISCUSSÃO}

Os municípios escolhidos para analise abrangem uma área de 32.630 hectares, onde está inserida a área delimitada Parque Estadual do Jabre/PB, a região é frequentemente atingida por incêndios naturais e/ou causados por ações provenientes de atividades antrópicas, ainda assim, é possível encontrar nesse local espécies nativas, que se contrastam com os aspectos apresentados pela vegetação disposta nas áreas próximas a serra do Teixeira. Nas regiões serranas do Brasil, existe uma preocupação elevada com o uso e ocupação do solo, uma vez que são áreas em que o processo erosivo se dá de forma acelerada, devido à união do fator antrópico aos demais fatores influenciadores (topográfico, tipo do solo e cobertura vegetal).

O fator topográfico em regiões serranas apresenta elevada relevância visto a suscetibilidade frente à dinâmica dos processos que ocorrem nessas regiões. A declividade é um atributo primário calculado diretamente a partir do Modelo Digital de Elevação, dessa forma, as imagens SRTM foram importadas no software Qgis 2.18 e com a ferramenta 'analise do terreno' se calculou a declividade (Figura 2), o valor da inclinação foi calculado em porcentagem e dividida em 5 (cinco) classes de acordo com o que determina a Empresa Brasileira de Pesquisa e Pecuária (EMBRAPA).

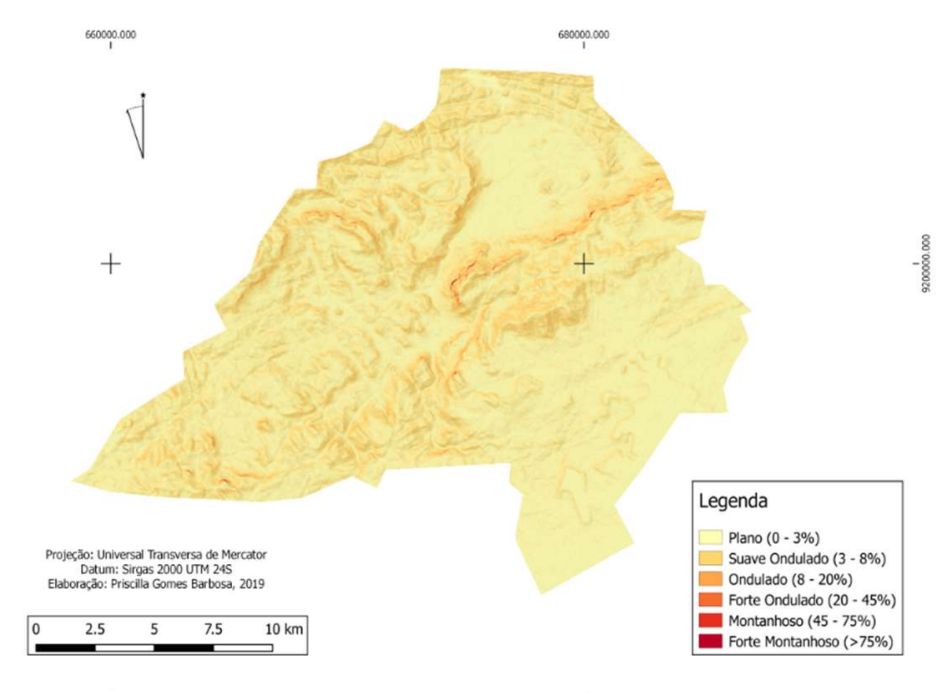

Figura 2: Espacialização do atributo declividade.

As classes de declividade visualizadas variam entre o plano e o forte montanhoso, e denota uma grande influência em relação a diversos fatores, como: escoamento superficial que sugere a atenuação de processos de erosão do solo. Sendo, portanto, a determinação da declividade uma forma de representação quantitativa do comportamento espacial do relevo, orientando o cumprimento da legislação ambiental em termos ambientais, como também dispor sobre o planejamento das intervenções antrópicas no ambiente.

No mapa hipsmétrico (Figura 3), são apresentadas as diferentes cotas distribuídas na região de estudo, com elevação mais baixa variando entre 0-64m e a mais alta $>185 \mathrm{~m}$, sendo esta última predominante 
na maior parte do território. A distribuição das cores na visualização espacial obedece aos aspectos altimétricos identificados no mapa, à coloração vermelha representa as regiões montanhosas e berços de nascentes, as demais cores estão associadas a elementos como: topo de morro, elevações próximas a canais de drenagem, encostas e canais de médio curso (FURLAN et al., 2018).

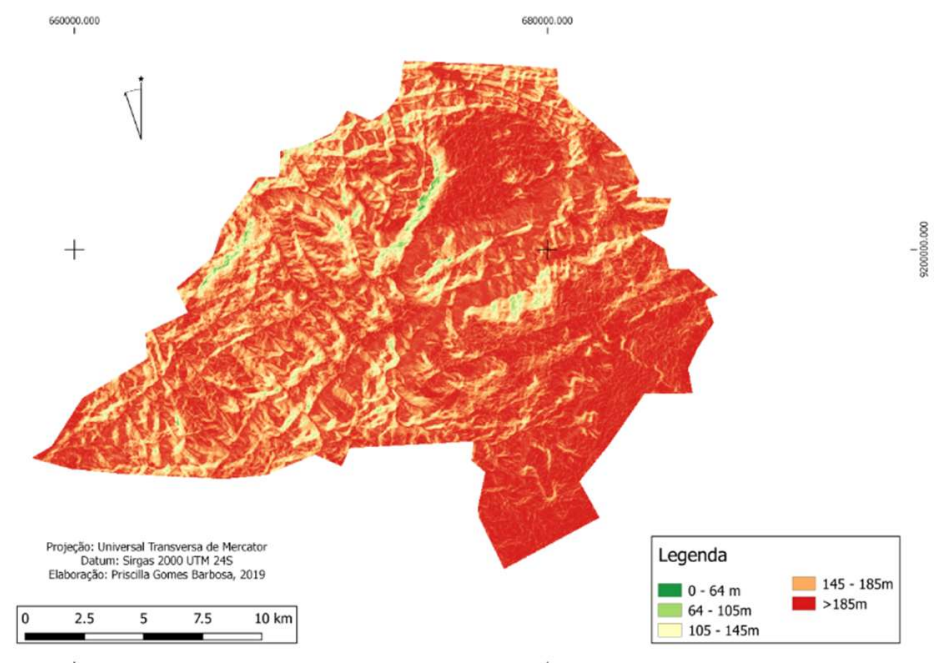

Figura 3: Espacialização do atributo hipsometria.

As cinco classes hipsometricas adotadas são representativas na definição da distribuição altimétrica, indicando as áreas em que predominam menores altitudes e as áreas em que predominam as maiores altitudes, ou seja, informações que entre outros aspectos podem auxiliar na elaboração de planos de mitigação a processos erosivos, bem como de monitoramento de áreas de encosta e/ou vulneráveis.
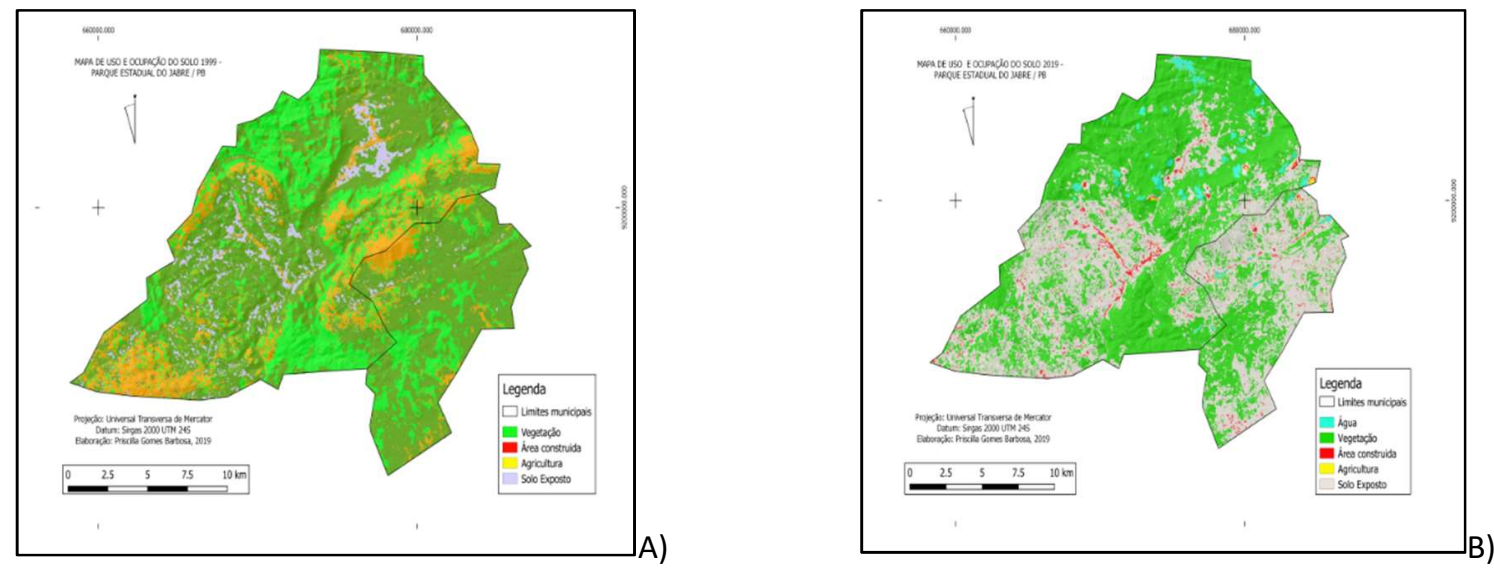

Figura 4: Espacialização do uso e ocupação do solo 1999a (Landsat 4-5) e 2019b (Sentinel 2).

A ocupação do espaço nesta região pelas atividades antrópicas ocorreu de forma desordenada e sem planejamento, a urbanização deste território acarretou na destruição de matas ciliares, contaminação de rios e outros aspectos que influenciaram diretamente na qualidade ambiental, como também na qualidade de vida da população inserida no contexto. O Parque Estadual Pico do Jabre, um importante elemento da paisagem dessa região criado em 1992, não possui plano de manejo local (obrigatório para áreas de conservação) segundo exige o decreto no 4.340/2002 e na Lei no 9.985/2000. Nesse sentindo, a criação de 
dados ambientais que possam auxiliar nesse processo se torna um elemento imprescindível. A Figura 4, apresenta as alterações do uso e ocupação territorial no período de 3 décadas sob a perspectiva da geotecnologia.

Com o objetivo de compreender as alterações no uso e ocupação do solo na região do Parque Estadual do Jabre se realizou o mapeamento da área, classificando as atividades praticadas com o auxílio do plugin dzetesaka via Qgis 2.18. Para esta analise utilizou-se imagem multiespectral dos satélites Landsat 4-5 (1999) e Sentinel 2 (2019), disponível gratuitamente na base de dados da USGS (United States Geological Survey - Levantamento Geológico dos Estados Unidos). Os alvos foram definidos através de variáveis numéricas e saídas (classes) nominais, Tabela 2.

Tabela 2: Alvos e classes para a composição do mapa de uso e ocupação do solo.

\begin{tabular}{|l|l|}
\hline Alvo & Classes \\
\hline 1 & Água \\
\hline 2 & Vegetação \\
\hline 3 & Área construída \\
\hline 4 & Agricultura \\
\hline 5 & Solo Exposto \\
\hline
\end{tabular}

A análise da imagem foi realizada através de interpretação visual. Este procedimento interpreta a imagem diretamente na tela do computador, utilizando elementos básicos de interpretação, como cor, textura, forma, tonalidade, tamanho, sombra, padrão, adjacências e localização geográfica (MAKAYA et al., 2019; SCHUSSEL et al., 2015). Comparando as imagens de 1999 e 2019 (Gráfico 1), observou-se um aumento da área de solo exposto e diminuição das áreas de vegetação, isto se dá entre outros fatores pelo regime pluviométrico da região semiárida e/ou atividades degradantes, naturais ou antrópicas que favorecem a ocorrência de solo desnudo. Quanto a vegetação, predominantemente arbustiva, foi possível observar que os maiores fragmentos dessa classe ocorrem nas regiões mais elevadas e próximas às margens dos rios. Foram visualizados ainda em menor ocorrência os elementos: curso d'água, urbanização e agricultura (com localização pontual).

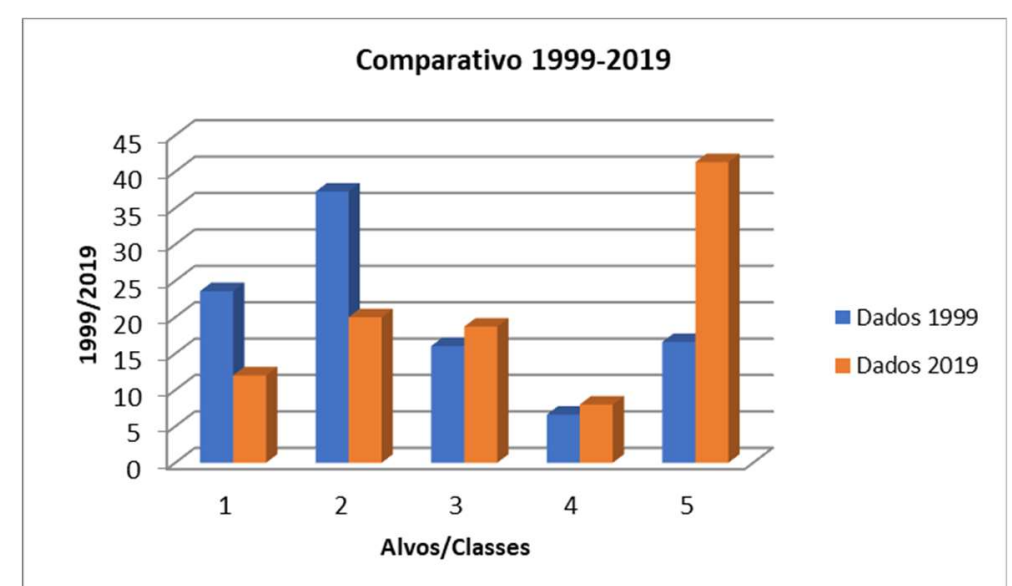

Gráfico 1: Gráfico comparativo entre as classes nos anos de 1999 e 2019.

Outro elemento importante no processo de classificação de imagens de satélite é o fator operador, 
etapa em que pode haver erros ou imprecisão na identificação dos elementos da imagem em virtude de fatores como: inexperiência do operador, alteração ou falhas nos pixels das imagens, erro de leitura do plugin, entre outros aspectos, demonstrando-se, portanto a necessidade do conhecimento (ao menos parcial) da área de estudo para realização de uma classificação mais próxima da realidade.

\section{CONCLUSÕES}

Por tudo isso, conclui-se que a área que compõe o Parque Estadual do Jabre, apresenta um estágio de uso e ocupação primário, visto a distribuição espacial das atividades de urbanização e agrícola. No entanto, observa-se a tendência de transformação na paisagem em virtude das características do meio e das necessidades antrópicas (econômicas e sociais) na região. Então, torna-se essencial que seja elaborado um plano de gestão ambiental para condicionar o uso e a conservação regional, sabendo que a área em questão tem grande contribuição no equilíbrio das variáveis ambientais e no desenvolvimento sustentável da população inserida nesse contexto.

Nesse sentido, é possível avaliar que a metodologia utilizada concebeu dados de análise de uso e ocupação do solo capazes de gerar informações sobre a área de estudo ao longo do período proposto, e embora não tenha contemplado as características quantitativas e qualitativas dos atributos morfométricos, comportaram-se, como instrumento útil na concepção de informações que poderão ser utilizadas como suporte na elaboração de políticas públicas de planejamento e gestão para a minimização dos riscos de uma ocupação inadequada do solo e a facilitação da adaptação as alterações climáticas.

A utilização de Geotecnologias e dados de sensoriamento remoto, integrados a Sistema de Informações Geográficas (SIG) possibilitou a espacialização das formas de ocupação do solo na região do Parque Estadual do Jabre/PB no intervalo de 30 anos (1999-2019) e apesar dos resultados não serem precisamente exatos, demonstrou-se como sendo uma ferramenta eficiente no apoio as tomadas de decisão no planejamento territorial regional. Dessa forma, o monitoramento continuo através de elementos geoecológicos pode servir como suporte na mitigação e prevenção de problemas ambientais causados por atividades antrópicas bem como por fatores naturais, além de serem facilitadores no cumprimento da legislação ambiental.

\section{REFERÊNCIAS}

AQUINO, A. R.; PALETTA, F. C.; ALMEIDA, J. R.

Vulnerabilidade ambiental. São Paulo: Blucher, 2017.

BÜNEMANN, E. K.; BONGIORNO, G.; BAI, Z.; CREAMER, R. E.; DEYN, G. D.; GOEDE, R.; FLESKENS, L.; GEISSEN, V.; KUYPER, T. W.; MADER, P.; PULLEMAN, M.; SUKKEL, W.; VAN GROENIGEN, J. W.; BRUSSAARD, L.. Soil quality: A critical review. Soil Biology and Biochemistry, v.120, p.105-125, 2018. DOI: https://doi.org/10.1016/j.soilbio.2018.01.030

CUIABANO, M. N.; NEVES, S. M. A. S.; NUNES, M. C. M.; SERAFIM, M. E.; NEVES, R. J.. Vulnerabilidade ambiental à erosão hídrica na sub-bacia do córrego do guanabara/reserva do Cabaçal/MT Brasil. Geociencias, v.36, n.1, p.138-153, 2017.

FERREIRA, A. M. R.; ROIG, H. L.; MAROTTA, G. S.; MENEZES, P. H. B. J.. Utilização de aeronaves remotamente pilotadas para extração de mosaico georreferenciado multiespectral e modelo digital de elevação de altíssima resolução espacial. In: SIMPÓSIO BRASILEIRO DE SENSORIAMENTO REMOTO, 16. Anais. Foz do Iguaçu: INPE, 2013.

FURLAN, A. R.; TRENTIN, R.; ROBAINA, L. C. S.. Classificação dos elementos do relevo a partir da metodologia dos geomorphons na bacia hidrográfica do rio Apuaê-Mirim, RS. 
Boletim Geográfico do Rio Grande do Sul, Porto Alegre, n.32, p.27-45, 2018.

GRECO, M. G.. Analisis socioambiental de espacios vulnerables sometidos a desastres, algunos estúdios de caso em el norte de la republica Argetina. In: ENCUENTRO DE GEÓGRAFOS DE AMÉRICA LATINA, 15. Anais. Havana, 2015.

MAKAYA, N.; DUBE, T.; SEUTLOALI, K.; SHOKO, C.; MUTANGA, O.; MASOCHA, M.. Geospatial assessment of soil erosion vulnerability in the upper uMgeni catchment in KwaZulu Natal, South Africa. Physics And Chemistry Of The Earth, Parts A/b/c, p.0-1, 2019. DOI: http://doi.org/10.1016/j.pce.2019.02.012

MEHRA, M.; SINGH, C. K.. Spatial analysis of soil resources in the Mewat district in the semiarid regions of Haryana, India. Environment, Development and Sustainability, v.20, n.2, p.661-680, 2018.

MIRHOSSEINI, M.; FARSHCHI, P.; NOROOZI, A. A.; SHARIAT, M.; AALESHEIKH, A. A.. Changing land use a threat to surface water quality: a vulnerability assessment approach in Zanjanroud Watershed, Central Iran 1. Water Resources, v.45, n.2, p.268-279, 2018.

NGUYEN, H. H.; RECKNAGEL, F.; MEYER, W.; FRIZENSCHAF,
J.; SHRESTHA, M. K.. Modelling the impacts of altered management practices, land use and climate changes on the water quality of the Millbrook catchment-reservoir system in South Australia. Journal of Environmental Management, v.202, p.1-11, 2017.

PEREIRA, R. A.; DANTAS NETO, J.. Efeito das atividades agropastoris sobre os atributos físico-químicos de três classes de solos de uma bacia hidrográfica no semiárido brasileiro. Boletim Goiano de Geografia, v.34, n.1, p.169188, 2014.

SCHUSSEL, Z.; NASCIMENTO NETO, P.. Gestão por bacias hidrográficas: do debate teórico à gestão municipal. Ambiente \& Sociedade, São Paulo, v.18, n.3, p.137-152, 2015.

SZLAFSTEIN, C.. Metodologia de análise e mapeamento de vulnerabilidade na Amazônia. In: CHANG, M.; GOÉS, K.; FERNANDES, L.; FREITAS, M. A. V.; ROSA, L. P..

Metodologias de estudos de vulnerabilidade à mudança de clima. Rio de Janeiro: ed. Interciência. p. 180-125. 2015.

ZHAO, J.; JI, G.; YUAN, T.; CHEN, Y.. Environmental vulnerability assessment for mainland China based on entropy method. Ecological Indicators, v.91, p.410-422, 2018.

A CBPC - Companhia Brasileira de Produção Científica (CNPJ: 11.221.422/0001-03) detém os direitos materiais desta publicação. Os direitos referem-se à publicação do trabalho em qualquer parte do mundo, incluindo os direitos às renovações, expansões e disseminações da contribuição, bem como outros direitos subsidiários. Todos os trabalhos publicados eletronicamente poderão posteriormente ser publicados em coletâneas impressas sob coordenação da Sustenere Publishing, da Companhia Brasileira de Produção Científica e seus parceiros autorizados. Os (as) autores (as) preservam os direitos autorais, mas não têm permissão para a publicação da contribuição em outro meio, impresso ou digital, em português ou em tradução. 